\title{
Research Article \\ Effect of Interface Layer Capacitance on Polydimethylsiloxane in Electrowetting-on-Dielectric Actuation
}

\author{
Shiraz Sohail, ${ }^{1}$ Soumen Das, ${ }^{2}$ and Karabi Biswas ${ }^{3}$ \\ ${ }^{1}$ Department of Electrical and Electronics Engineering, National Institute of Technology (NIT) Sikkim, Sikkim 737139, India \\ ${ }^{2}$ School of Medical Science and Technology, Indian Institute of Technology (IIT) Kharagpur, Kharagpur 721302, India \\ ${ }^{3}$ Department of Electrical Engineering, Indian Institute of Technology (IIT) Kharagpur, Kharagpur 721302, India
}

Correspondence should be addressed to Shiraz Sohail; s.sohail.in@ieee.org

Received 18 September 2014; Revised 3 December 2014; Accepted 3 December 2014

Academic Editor: Ahmed Ibrahim

Copyright (C) 2015 Shiraz Sohail et al. This is an open access article distributed under the Creative Commons Attribution License, which permits unrestricted use, distribution, and reproduction in any medium, provided the original work is properly cited.

\begin{abstract}
Electrowetting is an effective way to manipulate small volume of liquid in microfluidic applications. It has been sophisticatedly used in the fields of Lab-on-a-Chip (LoC) devices, optics, biomedical applications, and electronic paper (e-paper). Generally, YoungLippmann (Y-L) equation is used to relate the mechanical and electrical force involved in electrowetting-on-dielectric (EWOD) based actuation. And the general trend is to neglect the effect of double layer capacitance formed at the metal-liquid interface considering the Debye-length to be in the order of nanometer. But, at electrode-electrolyte-insulator interface, the effect of interface layer capacitance becomes significant and often leads to the mismatch between the experimental observation and theoretical result. In this work, the surface behaviour of polydimethylsiloxane (PDMS) for EWOD application is studied experimentally and a term " $k$ " has been introduced in the Y-L equation to match the theoretical and experimental result. Effect of interface layer capacitance has been observed in contact angle versus applied voltage experiment with different $\mathrm{pH}$ buffer solution. The introduction of " $k$ " term takes care of the interface layer capacitance which can not be neglected and plays a vital role when the applied electric potential is high.
\end{abstract}

\section{Introduction}

In recent times, fabrication and development of Lab-on-aChip (LoC) device is one of the most studied areas in different fields of science and engineering. The goal of $\mathrm{LoC}$ is to make a device in which medical diagnostics, environmental monitoring, and basic scientific research can be carried out in a rapid, low-cost, portable, and reliable environment [1]. These integrated microfluidic systems provide a number of benefits like ultrasensitive detection, lower diagnostic cost, reduced reagent consumption, higher levels of throughput, and reduced labor requirement. However, the major concern in microfluidic chip system is to control fluid effectively and accurately in microscale at either continuous or discrete flow process [2].

Several techniques have been developed to drive liquid using the concept of altering interfacial tension to produce an imbalance of forces inside the fluid [3]. Major surface tension based fluid dealing techniques are electrowetting (EW) [1], thermocapillarity-driven pumping [4], optoelectrowetting [5] based pumping, and vapor bubble-based pumping [6, 7].

Among these various mechanisms, electrowetting-ondielectric (EWOD) process is especially interesting due to benefits such as the absence of heat generation, low power consumption, and rapid switching response [3]. EW uses an externally applied electric field to manipulate small volumes of liquid by varying its interfacial tension with the surrounding and changing the macroscopic contact angle. It is an accurate means for actuating and controlling very small volume of liquid in microfluidic devices without the demand for any mechanical components $[8,9]$.

People have used different types of dielectric material to fabricate EWOD based devices [10, 11]. Among these materials, Teflon AF has very good hydrophobic characteristic but its high cost and low electric breakdown field make the material less attractive [12]. On the other hand, 
polydimethylsiloxane (PDMS) is extensively utilized as base material for EWOD phenomena, for its superior mechanical flexibility and biocompatibility $[8,10,13,14]$. It is a rubberlike hydrophobic material with comparatively high thermal stability $\left(>200^{\circ} \mathrm{C}\right)$, high electric breakdown field (typically $20 \mathrm{kV} / \mathrm{mm}$ ), and high initial contact angle of $\approx 107^{\circ}$. It also insures good insulation between the device and the living tissue [15]. Moreover, it is cheap and microstructures can be easily made by soft lithography technique $[16,17]$.

There has been progress in understanding the physics governing phenomena which influence EW systems. However, there is no consensus on the real cause of contact angle saturation. Kang's vertical force $[1,18]$ on the liquid interface near the triple line which increases quickly when the contact angle decreases could be an explanation for the phenomenon of the contact angle saturation. Verheijen and Prins [19] proposed a model of saturation based on the trapping of charges in the insulator when the voltage becomes sufficiently large. This trapping of charges reduces the density of charges at the solid-liquid interface and weakens the electrowetting effect. Their model included $V_{T}$ (potential at the level of the trapped charges on the gas side) which was difficult to estimate quantitatively. Peykov, Quinn, Ralston, and Sedev $[12,20]$ pioneered their model based on zero surface-liquid energy limit abbreviated as "PQRS model." The PQRS model considers that the saturation phenomenon is a thermodynamic limit of stability. The PQRS model predicts values of the saturation contact angle in reasonably good agreement with the experimental observations, although it sometime overestimates the contact angle. Shapiro and coworkers [1, 21,22] considered electric resistance of the liquid in their model. In their model, the droplet liquid is not perfectly conducting; it presents a small electric resistance that depends on the shape of the droplet. Shapiro's approach is very sensitive to the ratio of the resistivities of liquid droplet and dielectric. Other researchers have attributed saturation to fringe effect and ionisation of surrounding air as well. Modified Lippmann-Young law based on Langevin-Debye function shows good fit with the experimental results [1].

The researchers working in this field have carried out theoretical modeling of EWOD phenomena with different approaches neglecting electrical double layer capacitance considering Debye-length to be in nanometer range at metalliquid interface [1, 7, 9, 23-25]. In their work, YoungLippmann equation governing EWOD behavior is limited to small-medium electric potential and also leads to mismatch from experimental observation [1]. The novelty of this work is introduction of a " $k$ " term based on interface layer capacitance at the electrode-electrolyte-insulator interface in the classical Young-Lippmann equation which removes the mismatch between the experimental and theoretical results and also takes care of its application in high potential.

The present work studied the behavior of PDMS as dielectric as well as top hydrophobic layer for its use in EWOD based devices. It also observes the droplet morphology in terms of contact angle on wetting behavior of PDMS with electric potential and compared the experimental data from Young-Lippmann equation. Effect of interface layer capacitance has been noticed in contact angle versus applied

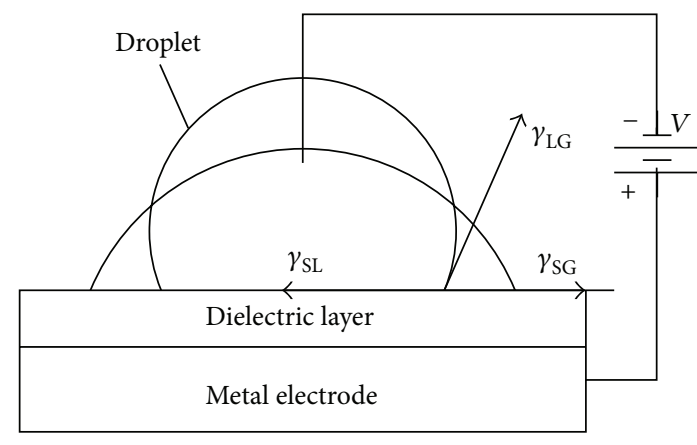

FIGURE 1: Basic configuration of open EWOD setup.

voltage experiment with different $\mathrm{pH}$ buffer solution. The experimental observation has been validated with theoretical simulation of Young-Lippmann equation with experimental parameter taking into consideration the interface layer behavior. Further, contact angle hysteresis and electrolysis phenomena have also been studied.

\section{Theory}

The basic mechanism of EWOD technique is shown in Figure 1. When a droplet is placed on top of the dielectric layer, it is rested by the force balance at three-phase contact line: the solid-gas $\gamma_{\mathrm{SG}}$, solid-liquid $\gamma_{\mathrm{SL}}$, and liquid-gas $\gamma_{\mathrm{LG}}$ interfacial surface tensions. At equilibrium state, the interfacial surface tensions balance is described by the Young equation $[23,25]$ :

$$
\gamma_{\mathrm{SG}}=\gamma_{\mathrm{SL}}+\gamma_{\mathrm{LG}} \cos (\theta),
$$

where $\theta$ is the contact angle at three-phase line. The simplest electrical equivalent model of the above setup is shown in Figure 2(a), where $C_{D}$ is the bulk insulator capacitance due to dielectric (PDMS) layer. This model neglects the effect of interface layer capacitance at the electrode-electrolyteinsulator interface. When electric potential $V$ is applied across the conducting droplet (as shown in Figure 1), ions and counterions rearrange themselves in the liquid and result in higher charge concentration at three-phase contact line [3]. This leads to decrease in surface energy at the solid-liquid interface and causes the droplet to locally spread out in open configuration of EWOD setup [7]. The relationship equation governing changes in $\gamma_{\mathrm{SL}}$ on application of electric potential $V$ is found by using stored energy in the system $E_{\text {sys }}$ and is expressed by Lippmann equation as given below [23]:

$$
\begin{gathered}
\gamma_{\mathrm{SL}}(V)=\gamma_{\mathrm{SL}}(0)-E_{\mathrm{sys}}, \\
E_{\text {sys }}=\frac{1}{2}\left(\frac{C_{D}}{A} V^{2}\right)=\frac{1}{2}\left(\frac{\epsilon_{0} \epsilon_{r}}{d} V^{2}\right),
\end{gathered}
$$

where $E_{\text {sys }}$ represents the system energy per unit area, $\epsilon_{0}$ is dielectric constant of vacuum, $\epsilon_{r}$ is dielectric constant of 


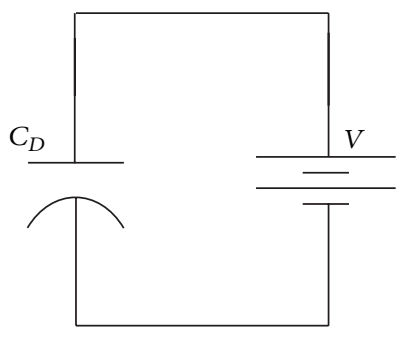

(a)

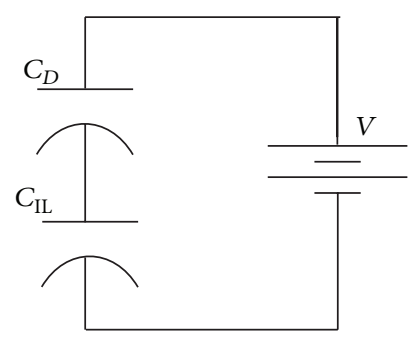

(b)

FIGURE 2: Equivalent circuit of the experimental setup: (a) without considering the interfacial layer capacitance, (b) considering interface layer capacitance $C_{\mathrm{IL}}$.

insulating layer, $d$ is the thickness of the dielectric material, and $A$ is the area under the droplet.

The well known Young-Lippmann (Y-L) equation [23] is obtained by combining (1) and (2) which expresses the relationship between the contact angle $\theta$ and the applied voltage $V$ and it is represented as given below:

$$
\begin{aligned}
& \cos \theta_{V}=\cos \theta_{0}+\frac{E_{\text {sys }}}{\gamma_{\mathrm{LG}}}, \\
& \text { hence, } \quad \theta_{V}=\cos ^{-1}\left(\cos \theta_{0}+\frac{E_{\mathrm{sys}}}{\gamma_{\mathrm{LG}}}\right) \text {, }
\end{aligned}
$$

where $\theta_{0}$ and $\theta_{V}$ are solid-liquid contact angles before and after application of electric potential, respectively.

The above derived Y-L equation is based on the classical thermodynamic approach $[1,23]$. Derivation of the Y-L equation can also be done by other approaches such as the energy minimization approach following the work of Shapiro and coworkers [1,21-23] and the electromechanical approach following the work of Jones et al. [24, 26], Kang [25], and recently Zeng and Korsmeyer [18]. All these approaches have different physical interpretations, although they all lead to the same classical Y-L equation.

The above treatment neglects the interfacial capacitance considering the Debye-length to be in the range of nanometer (nm) for metal-liquid interface [1]. But when an electrode is immersed in an electrolyte on a polymer surface, many complex electrochemical reactions take place at the electrodeelectrolyte-insulator interface. This is often described as the effect of double layer by the researchers and cannot be neglected in EWOD based device [12, 20, 27, 28]. Hence, the electrical equivalent circuit is modified as shown in Figure 2(b) including the interface layer effect. Here $C_{\mathrm{IL}}$ represents the interface layer capacitor. Since both the bulk capacitor and interface layer capacitor exist across either bulk of polymer or metal-liquid-polymer interface, the entire applied potential will fall across the bulk and interface. Hence, $E_{\text {sys }}$ will be modified to $E_{M \text { sys }}$ and is derived as follows:

$$
E_{M s y s}=\frac{1}{2}\left(\frac{C_{D} C_{\mathrm{IL}}}{C_{D}+C_{\mathrm{IL}}}\right) \frac{V^{2}}{A}=\frac{E_{\text {sys }}}{k},
$$

where $k=\left(C_{D} / C_{\mathrm{IL}}+1\right)$, which cannot be neglected for proper modeling of the actual EWOD based devices. Finally the modified solid-liquid contact angle expression on application of electric potential $V$ is given by the equation

$$
\theta_{V}=\cos ^{-1}\left(\cos \theta_{0}+\frac{E_{\text {sys }}}{k \times \gamma_{\mathrm{LG}}}\right) \text {. }
$$

The value of " $k$ " depends on the ratio of $C_{D} / C_{\mathrm{IL}}$ and is always greater than 1 . The " $k$ " term introduced in (5) for analytical expression of solid-liquid contact angle for a fixed applied potential implies that the value of $\theta_{V}$ becomes more due to interface layer effect than obtained from its general expression shown by (3). The actual voltage drop across the dielectric layer is less than applied voltage $V$ and thus the energy stored in the bulk capacitor $C_{D}$ will be reduced. As a result, the expected actuation voltage required to generate the effective force for the droplet movement will be more.

\section{Experiment}

Experiments have been carried out to explore the variation of contact angle with the change in applied potential in an open EWOD setup as shown in Figure 1 with PDMS as the dielectric layer on glass substrate. Different samples with varied thickness of the dielectric layer are prepared and subsequently contact angle has been measured by varying the applied voltage for these samples. Further, the reversibility and contact angle hysteresis phenomena on PDMS film have also been studied.

3.1. Sample Preparation. First of all, the glass substrate is dipped in piranha solution $\left(\mathrm{H}_{2} \mathrm{O}_{2}: \mathrm{H}_{2} \mathrm{SO}_{4}=1: 1\right)$ for $20 \mathrm{~min}$ and then cleaned thoroughly by acetone and DI water. Thereafter, the substrate is dried by $\mathrm{N}_{2}$ gun and heated in open air at $150^{\circ} \mathrm{C}$ for $30 \mathrm{~min}$. Aluminium thin film with thickness of $300 \mathrm{~nm}$ for making bottom electrode is deposited by thermal deposition (pressure: $1.5 \times 10^{-6} \mathrm{mbar}$, current: $2.96 \mathrm{~A}$, and time: $4.0 \mathrm{~min})$.

The dielectric layer has been prepared by thorough mixing of PDMS base (Sylgard 184 Silicone Elastomer Base, Dow Corning Corporation, USA) with its curing agent (Sylgard 184 Silicone Elastomer Curing Agent, Dow Corning Corporation, USA) in the 10:1 (w/w) ratio and degassed 


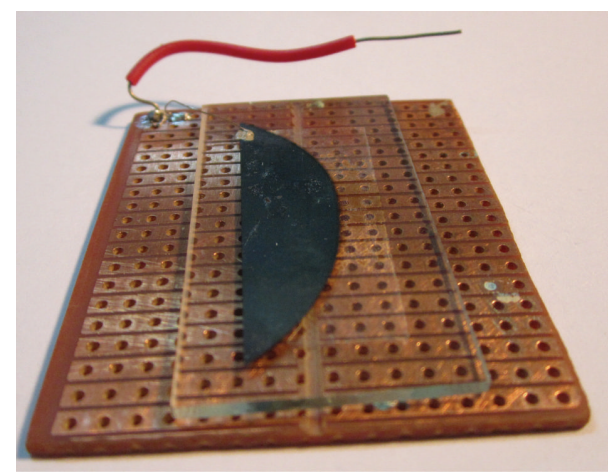

FIGURE 3: PDMS layer coated on aluminium metal electrode over glass substrate.

in vacuum for about 30 minutes to remove any trapped air-bubbles formed during mixing. Thereafter, PDMS has been spin-coated on the deposited metal aluminium layer by dispensing a few drops of mixture over the sample and spinning using a standard spin-coating machine (made by Laurell Technologies, model: WS-650). During this process, spin speed has been varied to achieve different thickness of dielectric PDMS layer on the top of metal coated glass sample. The samples have been baked at $80^{\circ} \mathrm{C}$ for 4 hours for complete polymerization of PDMS material.

To get different thickness of dielectric layer, glass substrates of dimension $2.5 \times 3.5 \mathrm{~cm}^{2}$ have been taken and coated with PDMS (on top of the metal electrodes) at different spin speed for 20-second spin time. Figure 3 shows photograph of a PDMS coated sample on aluminium electrode over glass substrate. The thickness of PDMS layer for all the samples has been precisely calculated by measuring the step height of the coated layer using NCS Profilometer (model: TalysurfSeries 2, Taylor Hobson Ltd., UK). The measurement was repeated for 3-4 times at various locations and averaged for every sample. The measured film thickness has a deviation of $\pm 10 \mathrm{~nm}$ for all the samples.

3.2. Contact Angle Measurement. A contact angle measurement setup has been made by a high speed camera (model: avA1000-100gc, made by Basler Aviator) connected to macro zoom lens (model: Zoom 7000, made by Navitar), mounted on tripod for recording droplet contact angle during experiment. The camera is interfaced to computer by video recording software (StreamPix $5 \times 64$ ) for remote control. For carrying experiment PDMS coated samples are placed at a three-axis movable stage for spatial adjustment and a drop of conductive liquid of $5 \mu \mathrm{L}$ by volume is dispensed over the sample by a micropipette (made by Accupipet, model: T10). The droplet is surrounded by open air during the experiment. The sample is connected to DC voltage supply (made by Aplab, model: 7332 Medium Voltage DC Power Supply) for changing voltage across the droplet. A platinum wire (diameter: $0.0508 \mathrm{~mm}$, hard, $99.95 \%$ ) connected to ground terminal of voltage supply is dipped into the droplet by adjusting laboratory clamp stand. The aluminium

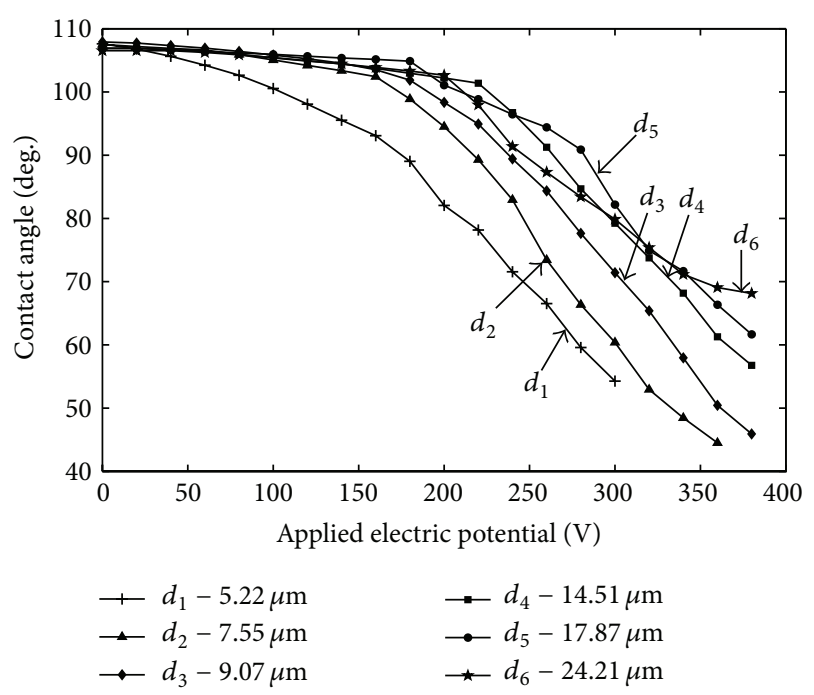

FIGURE 4: Variation of contact angle with increase in applied voltage for different samples having different thickness of PDMS coating. Here $d$ represents the thickness of PDMS layer.

electrode underneath the PDMS layer is connected to the other terminal of the voltage supply. The contact angle has been measured from the captured images by ImageJ analysis software. The conductive liquid used for experiment was a mixture of $1 \%$ potassium chloride $(\mathrm{KCl})$ in deionised water. The high speed camera is adjusted for recording side view of the droplet during the experiment. An illuminated circular area (LED light source) is created around the droplet, during the time of experiment, for clear picture. Each experiment was conducted on a fresh sample at $18^{\circ} \mathrm{C}$.

Six samples with different thickness of PDMS layer, $S_{1}(5.22 \mu \mathrm{m}), S_{2}(7.55 \mu \mathrm{m}), S_{3}(9.07 \mu \mathrm{m}), S_{4}(14.51 \mu \mathrm{m}), S_{5}$ $(17.87 \mu \mathrm{m})$, and $S_{6}(24.21 \mu \mathrm{m})$, with $\pm 10 \mathrm{~nm}$ deviation in thickness have been considered to experimentally verify the relation between contact angle $\theta$ and applied potential $V$. The experiment was performed with gradually increasing external electric potential in steps of $20 \mathrm{~V}$, starting from $0 \mathrm{~V}$ to $380 \mathrm{~V}$, and the images of the droplet were captured to measure the contact angle. To underline the effect of interface layer capacitance, properties of Debye layer have been modified [29] by using buffer solution of different $\mathrm{pH}$ values $(\mathrm{pH} 4$, $\mathrm{pH} 7$, and $\mathrm{pH}$ 9.2) for droplet, dispensed on fresh substrates of fixed thickness $(12 \mu \mathrm{m} \pm 50 \mathrm{~nm})$ for contact angle versus applied potential experiment. Subsequently, the contact angle versus applied electric potential for every sample has been plotted as shown in Figures 4 and 6. The indigenously developed experimental setup produces almost the same result as produced by standard goniometer.

\section{Results and Discussion}

From Figure 4, it is apparent that as the applied electric potential increases, the contact angle decreases and the trend is to follow Y-L equation for all the six samples $\left(S_{1}-S_{6}\right)$. 


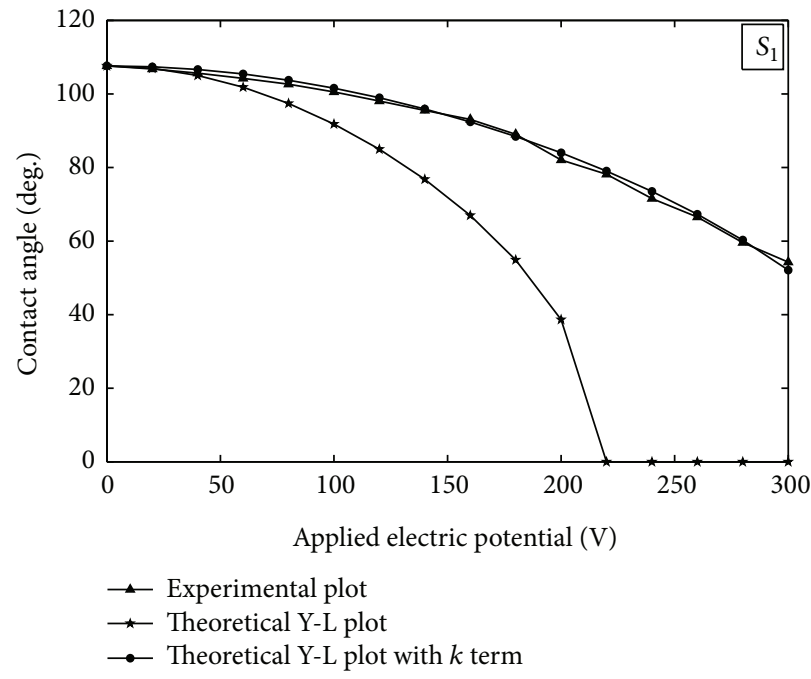

(a) Sample: $S_{1}$

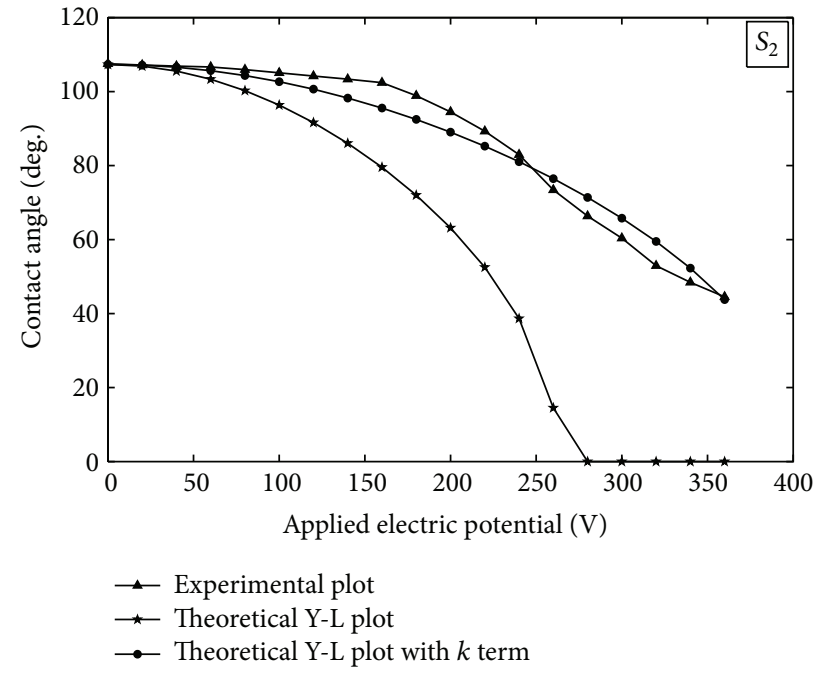

(b) Sample: $S_{2}$

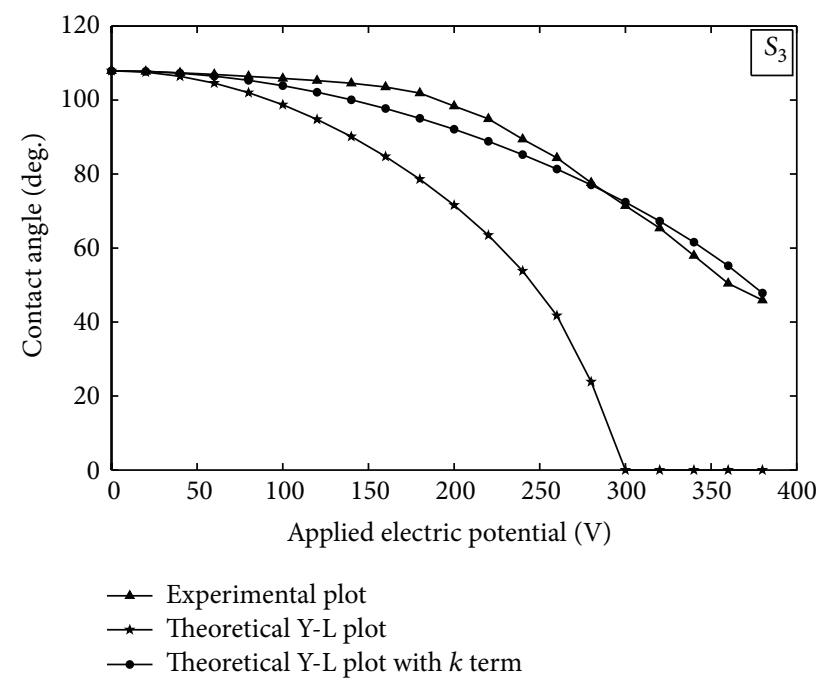

(c) Sample: $S_{3}$

FIGURE 5: Comparison between the data (experimental) curve, theoretical Y-L plot, and theoretical Y-L plot with " $k$ " term for samples $S_{1}, S_{2}$, and $S_{3}$.

The reason behind this decrease in contact angle is that as applied voltage increases, the electrostatic force due to capacitance formed between droplet and metal electrode increases leading to decrease in contact angle.

Among these six samples, three are taken $\left(S_{1}, S_{2}\right.$, and $S_{3}$ ) as representative case to verify the Y-L equation, both theoretically and experimentally. The data curves obtained through experiments and Y-L plots obtained through theoretical simulation have been shown in Figure 5. It can be seen from the figure that there is a considerable mismatch between Y-L plots governing EWOD behaviour and data curves for every sample. This mismatch increases as the supply voltage increases. Most important observation is that, after certain applied potential on the $x$-axis, simulated Y-L plots converge to zero on the $y$-axis (i.e., zero contact angles) for every sample as shown in Figure 5. But the data curves have still nonzero values on the $Y$-axis and these go on decreasing. For example, Y-L plot for $S_{1}$ converges to zero on the $Y$ axis at $220 \mathrm{~V}$ but the data curve for this sample has a value of $82.17^{\circ}$ and it goes on decreasing up to $300 \mathrm{~V}$ of applied potential reflecting $54.27^{\circ}$ contact angle on the $Y$-axis. Similar observations have been found for other samples too.

Different researchers have reported the validity of Y-L equation in EWOD process for a limited range of electric potential $[1,20]$. It becomes apparent that, combining (2) and (3), the necessary condition obtained for the validity of Y-L equation for hydrophobic surface is as follows:

$$
\frac{2 d \gamma_{\mathrm{LG}}}{\epsilon_{0} \epsilon_{r}}\left(1+\cos \theta_{0}\right) \leq V^{2} \leq \frac{2 d \gamma_{\mathrm{LG}}}{\epsilon_{0} \epsilon_{r}}\left(1-\cos \theta_{0}\right)
$$




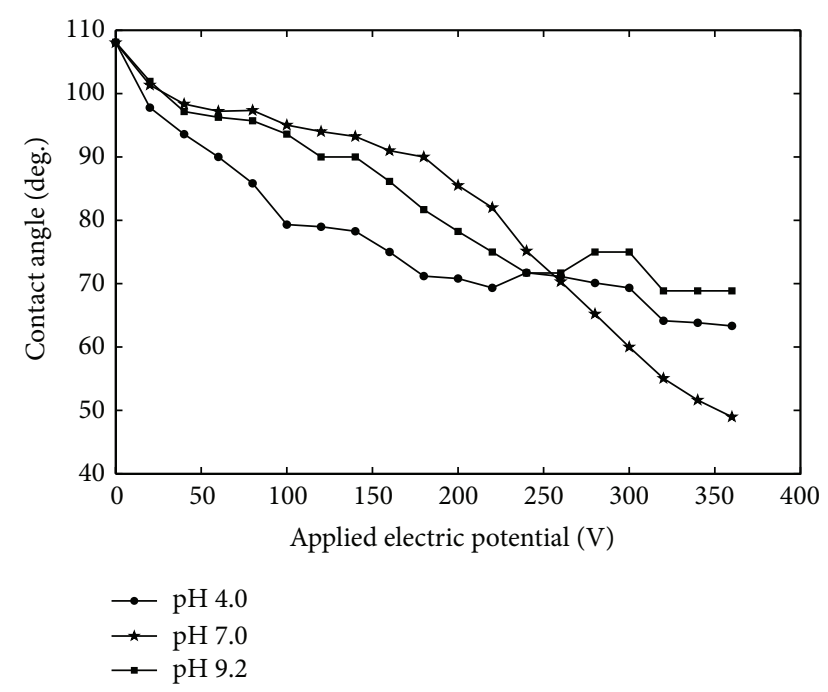

FIgURE 6: Contact angle versus applied voltage plot for fresh substrates of fixed thickness $(12 \mu \mathrm{m} \pm 50 \mathrm{~nm})$ with buffer solution of different $\mathrm{pH}$ values ( $\mathrm{pH} 4, \mathrm{pH}$, and $\mathrm{pH}$ 9.2).

The above condition fails if the total voltage drop " $V$ " in the system is considered only across the bulk capacitor $C_{D}$. The inclusion of interface layer capacitance $C_{\mathrm{IL}}$ and hence the voltage drop across the interface layer reduces the actual actuating voltage (the voltage across $C_{D}$ ). This is also reflected in Figure 5, where, after inclusion of " $k$ " term in Y-L equation, the data curves closely follow the theoretical Y-L plot for each sample. Significant observation is that the early convergence of Y-L plot has vanished for all samples.

The electrical double layer (EDL) represents the interface between a solid surface (polarized electrode) and an electrolyte. The charged surface attracts nearby counterions and repels coions present in the solution forming an electrical double layer capacitor. Effect of interface layer capacitance $C_{\mathrm{IL}}$ has been observed by modifying Debye layer properties with different $\mathrm{pH}$ buffer solution [29]. Figure 6 shows variation of contact angle with increase in applied voltage on fresh substrates of fixed thickness $(12 \mu \mathrm{m} \pm 50 \mathrm{~nm})$ with buffer solution of different $\mathrm{pH}$ values ( $\mathrm{pH} 4, \mathrm{pH} 7$, and $\mathrm{pH}$ 9.2). The different data curves in Figure 6 corresponding to different $\mathrm{pH}$ value underline the effect of $C_{\mathrm{IL}}$ because, in these cases, bulk capacitor $C_{D}$ is not changing due to fixed thickness of insulation layer. This dependence of EWOD phenomena on $\mathrm{pH}$ value of solution is in agreement with the work of Quinn et al. performed on Teflon AF 1600 developed by DuPont AF1600 with glass transition temperature $160^{\circ} \mathrm{C}$ and dielectric constant 1.93 [12].

The classical Poisson-Boltzmann (PB) theory of electrolytes assumes a dilute solution of point charges with meanfield electrostatic forces. This predicts large ion concentrations (exceeding close packing) for high surface potentials in the absence of surface reactions but in reality ions lose their classical Poisson-Boltzmann distribution due to crowding of ions near a surface, even if the bulk solution is very dilute.
Several modifications of the PB equation have been proposed to account for the finite size of ions in equilibrium [30].

Kilic et al. considered steric effects for canonical problem of charging in thin double layer at large applied voltage viewed as an effective circuit element, using two simple continuum models: (i) a "composite layer" model, consisting of a dilute $\mathrm{PB}$ outer region and, at high voltage, an inner "condensed layer" of ions packed at the steric limit and (ii) the modified Poisson-Boltzmann (MPB) equations [30]. The key results, common to both steric models, were as follows: (i) the differential capacitance is weaker at moderate and high potentials than if steric effects are neglected (as in the PB scheme); (ii) at moderate concentrations, the differential capacitance varies nonmonotonously with a peak at intermediate voltages and a slow decrease towards zero at higher voltages; (iii) at very high concentrations, the differential capacitance is a strictly decreasing function of voltage (in the MPB model) [30]. Inclusion of insulating surface layer between the metal electrode and the electrolyte leads to weakening of total capacitance in nonmonotonous fashion with a maximum at intermediate values and a further decrease to zero [30]. Hence, differential (and total) capacitance decreases at large voltage compared to classical PB theory.

The PB theory prediction of $C_{\mathrm{IL}}$ to be very high compared to $C_{D}$ had led researcher working in EWOD field to ignore the ratio of $C_{D} / C_{\mathrm{IL}}[1]$ and hence ignore the " $k$ " term in (4) whose value will always be greater than unity and varies with change in the ratio of $C_{D} / C_{\mathrm{IL}}$. But as evident from Figure 6 and the work of Kilic et al., $C_{\mathrm{IL}}$ is instrumental and at high potential decreases to a value that may lead to nonzero finite value of the ratio $C_{D} / C_{\mathrm{IL}}$.

Droplet solution, insulation layer, and electrode material had been kept the same in all the experiments mentioned above, to keep the properties of Debye layer unchanged. However, thickness of PDMS layer had been varied for changing the bulk capacitance. The $k$ term is a function of both interface layer and bulk capacitance (see (4)). But since interface layer capacitance would be the same for all samples (i.e., for $S_{1}, S_{2}, S_{3}, S_{4}, S_{5}$, and $S_{6}$ ), the value of $k$ should vary following a relation with thickness of insulation layer. The values of adjustable parameter " $k$ " for samples $S_{1}, S_{2}, S_{3}, S_{4}$, $S_{5}$, and $S_{6}$ are $2.66,2.38,2.3,1.9,1.75$, and 1.32, respectively. The variation in the value of " $k$ " with thickness of the PDMS layer is shown in Figure 7. From the values of " $k$ " chosen for the best fit, it is observed that its value increases as the thickness of dielectric layer for samples decreases. Since $C_{D}$ is modelled as parallel plate capacitor and is connected in series with $C_{\mathrm{IL}}$, theoretically " $k$ " is inversely related to $d$. This measure agrees with the values of " $k$ " term obtained from data curves for best fit. $C_{\mathrm{IL}}$ is dominated by bulk capacitance if very thick insulation layer is used. But again it is not desirable in order to reduce the actuation voltage. So finally a tradeoff is required between actuation voltage and the mismatch in Young-Lippmann equation. It is worth mentioning here that if the effect of $C_{\mathrm{IL}}$ is neglected considering $C_{\mathrm{IL}} \gg C_{D}$, then " $k$ " gets cancelled out but that results in a mismatch between Y-L plots and data curves as shown in Figure 5. Hence it can be asserted that $C_{\mathrm{IL}}$ has major and definite role 


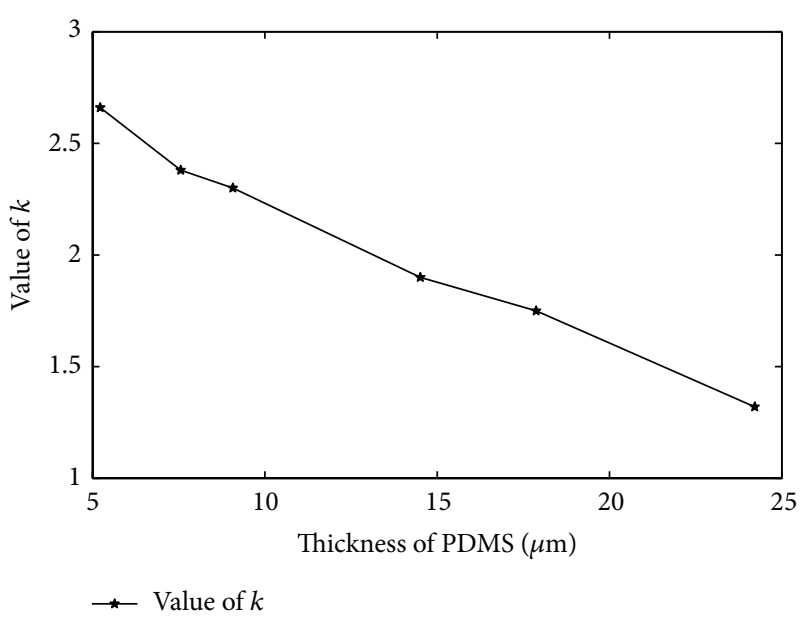

FIGURE 7: Variation of the value of " $k$ " with the thickness of the PDMS layer.

in EWOD process and contributes " $k$ " term which removes the mismatch.

The interface layer capacitance at the electrodeelectrolyte-insulator depends on several parameters such as electrode material (metals, electrode porosity, and the presence of layers of either oxides or polymeric films or other solid materials at the surface), type of electrolyte, extent of specific adsorption of ions and molecules, and electrolyte temperature [31]. It also depends on mixing ratio of PDMS base with its curing agent, vacuum treatment during degassing, sample cleaning, annealing temperature, and time [32]. Further study is required for measurement of $C_{\mathrm{IL}}$ to calculate exact value of " $k$ " term and better physical insight into the EWOD process.

To study the effect of hysteresis and reversible character of PDMS film, the applied electric potential has been decreased back to $0 \mathrm{~V}$. For all samples, contact angle at zero potential during decrease of applied potential is less than that during increase of applied potential leading to actuation hysteresis as shown in Figure 8 (two out of six-thinnest " $S_{1}$ " and thickest " $S_{6}$ " PDMS film - are provided for better readability).

Contact angle hystereses are due to the surface roughness, polymer-solution layer interaction, and the trapped charges in or between the insulation layers. As a result, high surface roughness and high polymer-solution interaction lead to more hystereses. The hysteresis effect decreases as the film becomes thinner. Electrostatic energy is trapped in the films and when the voltage is decreased, the stored energy is released. If the film is thicker, more energy is trapped inside the system which gives more hysteresis [33], which is apparent from Figure 8.

The photograph of Figure 9 shows the nature of the droplet and contact angle for sample $S_{1}$ on increasing electrical potential across the droplet. It has been observed in Figure 4 that initially there is a small variation in contact angle with increase in applied potential for the samples $S_{2}$, $S_{3}, S_{4}, S_{5}$, and $S_{6}$. Thereafter, the change in contact angle of

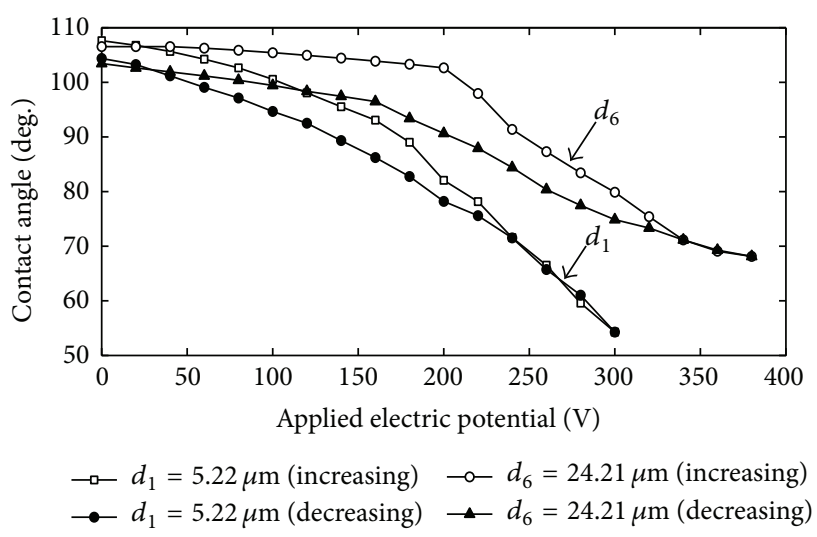

FIGURE 8: Hysteresis plot of contact angle with applied voltage for samples $S_{1}, S_{2}$, and $S_{3}$. Here $d$ represents the thickness of PDMS layer.

droplet becomes very prominent (e.g., $220 \mathrm{~V}$ for samples $S_{5}$ and $S_{6}$ ). Several definitions of threshold voltage are found in literature $[1,10,12,19,23,34-37]$. In the present work, threshold voltage is considered as the applied potential at which prominent change in contact angle has been observed from experimental data in open EWOD setup. The relation between the thickness of the PDMS layer and the threshold voltage can be studied from Figures 4 and 8 . It is apparent that, for most of the cases, the threshold voltage for different samples increases as the thickness of the dielectric layer increases (i.e., lower thickness of the dielectric material is better for actuation). However, irregularity is found in few of the measured data in Figure 4 where lower film thickness has slightly higher threshold voltage than the thicker PDMS film. This may be attributed to the experimental error contributed from the used instruments and also the change in PDMS film property due to surface contamination.

\section{Conclusion}

Surface behavior of polydimethylsiloxane (PDMS) on electrical activation in electrowetting-on-dielectric (EWOD) and its verification with Young-Lippmann (Y-L) equation have been carried out experimentally in this paper.

The study raises an essential consideration during selection of dielectric material to make EWOD based devices. Generally, dielectric and hydrophobic properties are looked for, choosing the material of thin film on electrode without giving much attention to interface layer capacitance $C_{\mathrm{IL}}$ at the electrode-electrolyte-insulator interface. The presence of interface layer capacitance has been identified as one of the underlying causes behind the mismatch between the experimental and theoretical results in voltage versus contact angle experiment. A " $k$ " term has been introduced in the classical Y-L equation which takes care of the interface layer capacitance and removes the mismatch in the saturation region. Therefore, along with the dielectric and hydrophobic properties, parameters and film material properties affecting 


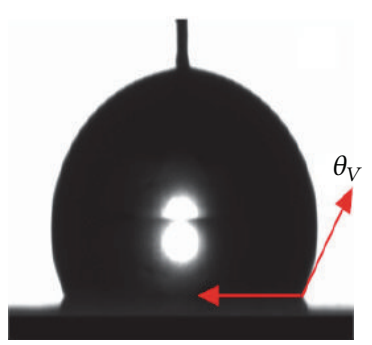

(a)

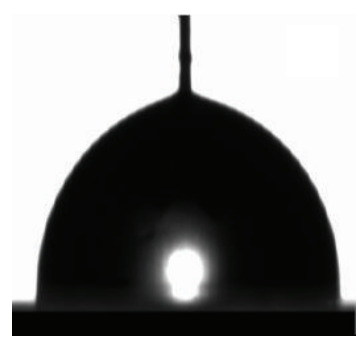

(c)

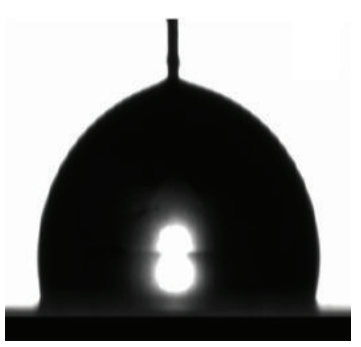

(b)

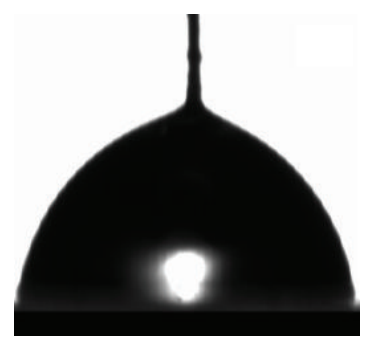

(d)

FIGURE 9: Photograph of the droplet on sample $S_{1}$ with change in applied voltage: (a) $\theta_{0}=107.62^{\circ}$; (b) $\theta_{V}=98.27^{\circ}$; (c) $\theta_{V}=87.37^{\circ}$; (d) $\theta_{V}=62.32^{\circ}$.

interface layer capacitance $C_{\mathrm{IL}}$ should also be considered during selection of insulation layer in EWOD actuation.

\section{Conflict of Interests}

The authors declare that there is no conflict of interests regarding the publication of this paper.

\section{References}

[1] J. Berthier, Microdrops and Digital Microfluidics, William Andrew, 2008.

[2] L. Li, H. Hu, H. Lin, and D.-t. Ye, "Electrowetting of the blood droplet on the hydrophobic film of the EWOD chips," in Proceedings of the 27th Annual International Conference of the Engineering in Medicine and Biology Society (IEEE-EMBS '05), pp. 1941-1944, Shanghai, China, 2005.

[3] A. Arzpeyma, S. Bhaseen, A. Dolatabadi, and P. WoodAdams, "A coupled electro-hydrodynamic numerical modeling of droplet actuation by electrowetting," Colloids and Surfaces A: Physicochemical and Engineering Aspects, vol. 323, no. 1-3, pp. 28-35, 2008.

[4] T. S. Sammarco and M. A. Burns, "Thermocapillary pumping of discrete drops in microfabricated analysis devices," AIChE Journal, vol. 45, pp. 350-356, 1999.

[5] P. Y. Chiou, H. Moon, H. Toshiyoshi, C.-J. Kim, and M. C. Wu, "Light actuation of liquid by optoelectrowetting," Sensors and Actuators A: Physical, vol. 104, no. 3, pp. 222-228, 2003.

[6] X. Geng, H. Yuan, H. N. Oguz, and A. Prosperetti, "Bubblebased micropump for electrically conducting liquids," Journal of Micromechanics and Microengineering, vol. 11, no. 3, pp. 270276, 2001.

[7] V. Bahadur and S. V. Garimella, "An energy-based model for electrowetting-induced droplet actuation," Journal of Micromechanics and Microengineering, vol. 16, no. 8, pp. 1494-1503, 2006.
[8] J. S. Kuo, P. Spicar-Mihalic, I. Rodriguez, and D. T. Chiu, "Electrowetting-induced droplet movement in an immiscible medium," Langmuir, vol. 19, no. 2, pp. 250-255, 2003.

[9] V. Bahadur and S. V. Garimella, "Electrowetting-based control of droplet transition and morphology on artificially microstructured surfaces," Langmuir, vol. 24, no. 15, pp. 8338-8345, 2008.

[10] H. Liu, S. Dharmatilleke, D. K. Maurya, and A. A. O. Tay, "Dielectric materials for electrowetting-on-dielectric actuation," Microsystem Technologies, vol. 16, no. 3, pp. 449-460, 2010.

[11] H. Moon, S. K. Cho, R. L. Garrell, and C.-J. Kim, "Low voltage electrowetting-on-dielectric," Journal of Applied Physics, vol. 92, no. 7, pp. 4080-4087, 2002.

[12] A. Quinn, R. Sedev, and J. Ralston, "Influence of the electrical double layer in electrowetting," The Journal of Physical Chemistry B, vol. 107, no. 5, pp. 1163-1169, 2003.

[13] M. Im, D.-H. Kim, J.-H. Lee, J.-B. Yoon, and Y.-K. Choi, "Electrowetting on a polymer microlens array," Langmuir, vol. 26, no. 14, pp. 12443-12447, 2010.

[14] W. Satoh, M. Loughran, and H. Suzuki, "Microfluidic transport based on direct electrowetting," Journal of Applied Physics, vol. 96, no. 1, pp. 835-841, 2004.

[15] E. Berthier, E. W. K. Young, and D. Beebe, "Engineers are from PDMS-land, biologists are from polystyrenia," Lab on a Chip, vol. 12, no. 7, pp. 1224-1237, 2012.

[16] K. Y. Suh, J. Seong, A. Khademhosseini, P. E. Laibinis, and R. Langer, "A simple soft lithographic route to fabrication of poly(ethylene glycol) microstructures for protein and cell patterning," Biomaterials, vol. 25, no. 3, pp. 557-563, 2004.

[17] O. J. A. Schneller, S. T. Brittain, and G. M. Whitesides, "Fabrication of glassy carbon microstructures by soft lithography," Sensors and Actuators A: Physical, vol. 72, no. 2, pp. 125-139, 1999.

[18] J. Zeng and T. Korsmeyer, "Principles of droplet electrohydrodynamics for lab-on-a-chip," Lab on a Chip, vol. 4, no. 4, pp. 265-277, 2004. 
[19] H. J. J. Verheijen and M. W. J. Prins, "Reversible electrowetting and trapping of charge: model and experiments," Langmuir, vol. 15, no. 20, pp. 6616-6620, 1999.

[20] V. Peykov, A. Quinn, and J. Ralston, "Electrowetting: a model for contact-angle saturation," Colloid and Polymer Science, vol. 278, no. 8, pp. 789-793, 2000.

[21] B. Shapiro, H. Moon, R. Garell, and C. Kim, "Equilibrium behavior of sessile drops under surface tension, applied external fields, and material variations," Journal Applied Physics, vol. 93, pp. 5794-5811, 2003.

[22] N. Fortner and B. Shapiro, "Equilibrium and dynamic behavior of micro flows under electrically induced surface tension actuation forces," in Proceedings of the International Conference on MEMS, NANO and Smart Systems, pp. 197-202, July 2003.

[23] F. Mugele and J.-C. Baret, "Electrowetting: from basics to applications," Journal of Physics: Condensed Matter, vol. 17, no. 28, pp. R705-R774, 2005.

[24] T. B. Jones, "An electromechanical interpretation of electrowetting," Journal of Micromechanics and Microengineering, vol. 15, no. 6, pp. 1184-1187, 2005.

[25] K. H. Kang, "How electrostatic fields change contact angle in electrowetting," Langmuir, vol. 18, no. 26, pp. 10318-10322, 2002.

[26] T. Jones, J. Fowler, Y. S. Chang, and C.-J. Kim, "Frequencybased relationship of electrowetting and dielectrophoretic liquid microactuation," Langmuir, vol. 19, pp. 7646-7651, 2003.

[27] F. Garbassi, M. Morra, and E. Ochiello, Polymer Surfaces, John Wiley \& Sons, 1994.

[28] R. Zimmermann, S. Dukhin, and C. Werner, "Electrokinetic measurements reveal interracial charge at polymer films caused by simple electrolyte ions," The Journal of Physical Chemistry B, vol. 105, no. 36, pp. 8544-8549, 2001.

[29] Y. Zhou and R. M. Raphael, "Solution pH alters mechanical and electrical properties of phosphatidylcholine membranes: relation between interfacial electrostatics, intramembrane potential, and bending elasticity," Biophysical Journal, vol. 92, pp. 2451-2462, 2007.

[30] M. S. Kilic, M. Z. Bazant, and A. Ajdari, "Steric effects in the dynamics of electrolytes at large applied voltages. II. Modified Poisson-Nernst-Planck equations," Physical Review E, vol. 75, no. 2, Article ID 021503, 2007.

[31] Z. Stojek, The Electrical Double Layer and Its Structure, vol. 2, Springer, Berlin, Germany, 2010.

[32] T. K. Kim, J. K. Kim, and O. C. Jeong, "Measurement of nonlinear mechanical properties of PDMS elastomer," Microelectronic Engineering, vol. 88, no. 8, pp. 1982-1985, 2011.

[33] K. Chakrabarty and J. Zeng, Design Automation Methods and Tools for Microfluidics Based Biochips, Springer, Berlin, Germany, 2006.

[34] J.-H. Chang, D.-Y. Choi, X. You, J. J. Pak, and S. Han, "Low voltage electrowetting on atomic-layer-deposited aluminum oxide," in Proceedings of the 5th IEEE International Conference on Nano/Micro Engineered and Molecular Systems (NEMS '10), pp. 612-615, Xiamen, China, January 2010.

[35] M. Vallet, M. Vallade, and B. Berge, "Limiting phenomena for the spreading of water on polymer films by electrowetting," The European Physical Journal B, vol. 11, no. 4, pp. 583-591, 1999.

[36] B. Berge and J. Peseux, "Variable focal lens controlled by an external voltage: an application of electrowetting," The European Physical Journal E, vol. 3, no. 2, pp. 159-163, 2000.

[37] F. Saeki, J. Baum, H. Moon, J.-Y. Yoon, C.-J. C. Kim, and R. L. Garrell, "Electrowetting on dielectrics (EWOD): reducing voltage requirements for microfluidics," Polymeric Materials: Science and Engineering, vol. 85, pp. 12-13, 2001. 

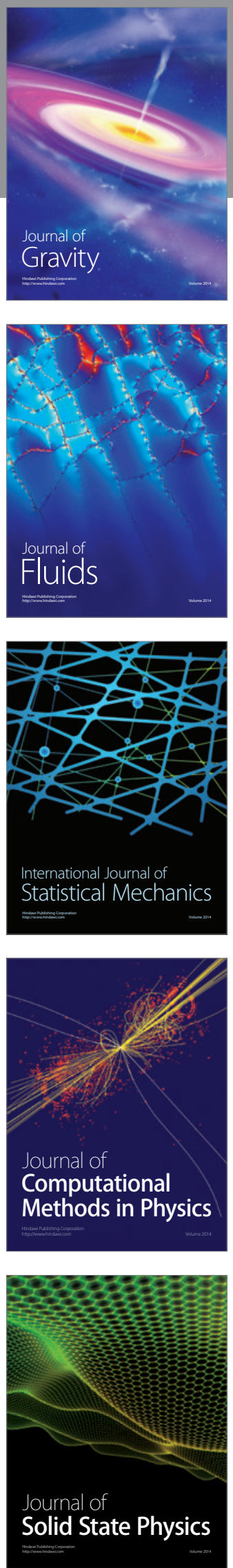

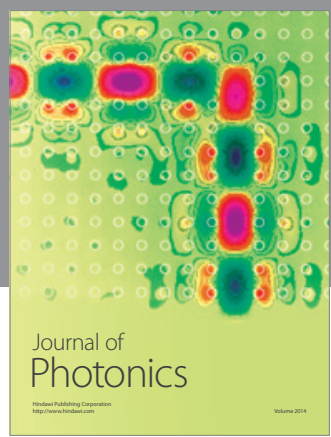

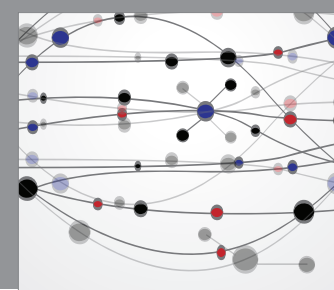

The Scientific World Journal

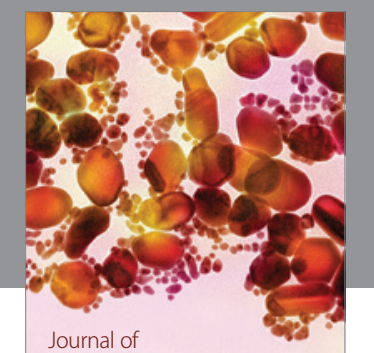

Soft Matter
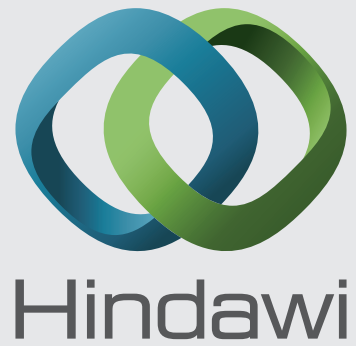

Submit your manuscripts at

http://www.hindawi.com
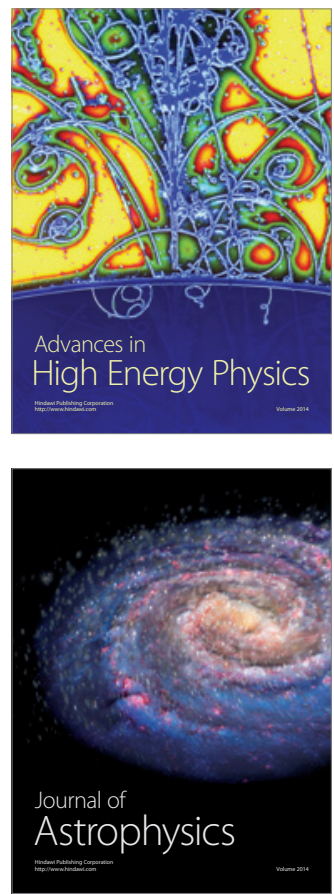
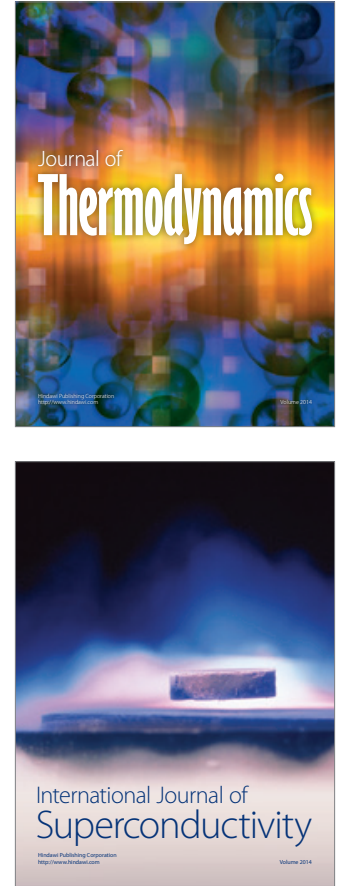
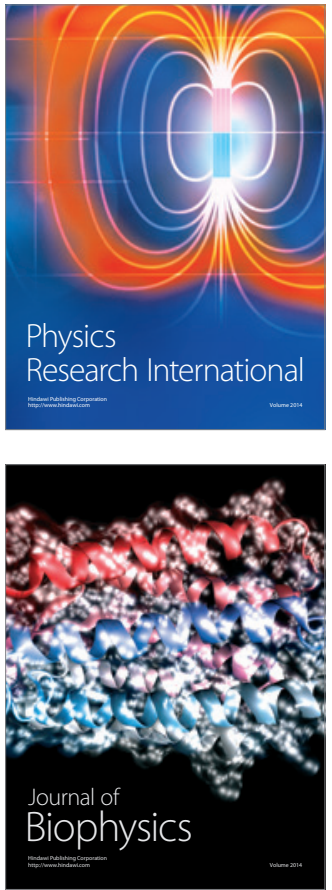
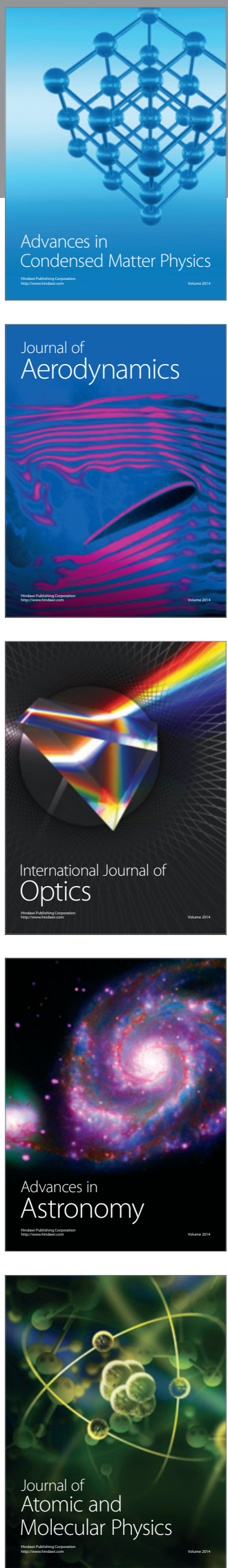